\title{
Children Persist Less When Adults Take Over
}

Julia A. Leonard*1, Dominique N. Martinez ${ }^{1}$, Samantha C. Dashineau ${ }^{1}$, Anne T. Park ${ }^{1}, \&$ Allyson P. Mackey ${ }^{1}$

\author{
Affiliations: \\ ${ }^{1}$ Department of Psychology, University of Pennsylvania, Levin Building, 425 S. University \\ Ave., Philadelphia, PA 19104 \\ *Corresponding author: Julia Leonard, email: jlnrd@sas.upenn.edu
}

\section{Acknowledgements}

We thank Jasmine Forde, Ursula Tooley, Austin Boroshok, Dee Asaah, and members of the Changing Brain Lab for help with data collection and coding, the Please Touch Museum for their partnership, participating families, and Angela Duckworth, Laura Schulz, and Elizabeth Brannon for critical feedback.

\section{Author Contributions}

J. A. Leonard and A. P. Mackey designed the study and wrote the manuscript. J. A. Leonard analyzed the data under the supervision of A. P. Mackey. J. A. Leonard, D. N. Martinez, S. C. Dashineau, and A. T. Park collected the data. A. T. Park provided critical revisions. All authors approved the final version of the manuscript for submission.

\section{Funding}

This research was supported by a Jacobs Foundation Early Career Research Fellowship to A.P.M. and a University of Pennsylvania MindCORE Postdoctoral Fellowship to J.A.L.

\section{Competing interests}

The authors declare no competing interests

Data and code availability

All data, materials, and preregistrations are available at https://osf.io/j52sf/. 


\title{
Children Persist Less When Adults Take Over
}

\begin{abstract}
Children need to learn to persist through challenges, yet adults sometimes step in to solve problems for them. Here, we looked at how adult taking over related to children's persistence. In an observational study ( $N=34$, ages $4-8)$, we found that parents who took over more often during a challenging puzzle task rated their children as dispositionally less persistent. To establish whether taking over can cause reduced persistence, we ran two preregistered experiments $(N=150$, ages 4-5). Children assigned to a taking over condition persisted less on a subsequent task compared to those in a teaching or a baseline condition. Reframing the context did not ameliorate the negative impact of taking over. The results suggest that taking over impairs children's persistence.
\end{abstract}

Keywords: Motivation, persistence, cognition, early childhood, parenting, social learning 


\section{Introduction}

The ability to persist through challenges has consequences for everything from learning in school, to succeeding at work, to maintaining social connections (Eskreis-Winkler, Shulman, Beal, \& Duckworth, 2014; Gottfried, 1985; Mischel, Shoda, \& Rodriguez, 1989). Individual differences in persistent behavior correlate with later academic and cognitive outcomes (Banerjee \& Tamis-LeMonda, 2007; Martin, Ryan, \& Brooks-Gunn, 2013; Messer et al., 1986; Mokrova et al., 2013), but we know relatively little about how to foster persistent behavior in young children. Here, we examine how caregiver and adult behavior influence effortful behavior in young children, with the goal of providing specific, concrete suggestions for how adults can promote children's persistence.

In correlational work with infants and toddlers in Western cultures, parenting that is supportive (e.g., praise, smiling), promotes autonomy (e.g., verbal feedback, helpful scaffolding, responsive to child direction), and uses process praise (e.g., praising effort, not ability) is related to children's current and future task persistence up to one year later (Deater-Deckard et al., 2006; Frodi, Bridges, \& Grolnick, 1985; Kelley, Brownell, \& Campbell, 2000; Lucca, Horton, \& Sommerville, 2019; Prendergast \& MacPhee, 2018; Yarrow et al., 1982). This form of supportive parenting is theorized to encourage children's persistence by fostering the development of self-efficacy and a sense of competence. Parenting that is intrusive and controlling (e.g., giving many directives without letting the child take independent action, completing tasks for the child), on the other hand, has been negatively related to young children's state persistence (Mokrova et al., 2012), potentially through undermining children's feelings of competence and autonomy. 
Experimental work has also shown that adults' actions and words can shape young children's persistence in a given moment. For example, infants make more attempts to activate a toy after watching an experimenter effortfully succeed in reaching her goals on two different toys (Leonard, Lee, \& Schulz, 2017). Similarly, preschoolers spend more time trying to open a secretly impossible box after watching an experimenter put in effort to open a similar box (Leonard, Garcia, \& Schulz, 2019; see also Zimmerman \& Blotner, 1979). This effect is amplified when the adult also states aloud that she values hard work (Leonard et al., 2019). When adults praise children for their effort ("process praise"), children consistently persist longer than when they are praised for their ability ("person praise") (Cimpian, Arce, Markman, \& Dweck, 2007; Kamins \& Dweck, 1999; Mueller \& Dweck, 1998).

However, correlational and experimental research in this area rarely overlap to create a full picture of how adult practices causally impact children's persistence. Correlational studies have identified which parenting practices relate to state persistence but leave open the question of causality (a particular concern because persistence is somewhat heritable; Deater-Deckard et al., 2006). Moreover, correlational work also tends to aggregate specific parenting behaviors into larger constructs, making it hard to know exactly which parenting behaviors relate to children's persistence. Experimental work on adult language and actions shows causality, but may not reflect common parenting practices. For example, in an observational study, only $3 \%$ of parents' child-directed speech included child-directed praise (Gunderson et al., 2013). Furthermore, past work on the relationship between parenting and children's persistence is often confounded with children's IQ. In many studies, children's persistence is measured as their time spent working on a challenging, but possible, cognitive task, like shape sorting (e.g., Frodi et al., 1985; Lucca et al., 2019; Mokrova et al., 2012). Individual differences in children's IQ may influence not only 
their ability to succeed on, but also their interest in and approach towards, these tasks. This leaves open the question of whether prior studies showing correlations between parenting behaviors and children's persistence may actually be capturing relationships between parenting and cognitive development more broadly.

In the current investigation, we bring together correlational and experimental approaches to look at how common parenting behaviors causally shape children's trait and state persistence irrespective of IQ. In an exploratory first study, we ask which parenting practices, elicited during a parent-child interaction task involving challenging puzzles, correlate with trait persistence in a socially and economically diverse sample of 4- to 8-year-old children. In a second, preregistered, random assignment experiment, we then test the causal direction of our correlational results from Study 1 in an independent sample of 4- to 5-year-old children. We chose to focus on children who are about to enter or just beginning formal education to probe how adult behavior influences children's persistence when they are learning many formative skills.

To capture children's persistent behavior, we take a two-pronged approach. In Study 1 we measure children's persistence with parent report, which quantifies persistent behavior across contexts, to explore how parenting behaviors relate to children's global trait persistence, controlling for children's IQ. In Studies 2 and 3, we measure persistence as children's time spent trying to do a secretly impossible task, to ask how short-term manipulations change children's state persistent behavior in that moment. This approach allows us to investigate whether parenting behaviors that correlate with children's trait persistence also causally impact children's state persistence. 


\section{Study 1}

\section{Methods}

The Institutional Review Board at [Institution taken out for blind review] approved Study 1, 2, and 3. Parents provided informed, written consent for their children's participation. Children age 8 gave written assent. Children younger than 8 verbally assented to participation.

Participants. Participants were recruited as part of a larger neuroimaging study through advertisements on public transportation, partnerships with local schools, outreach programs, and social media ads. Our study focused on 34 children (19 female) who had usable data on both relevant measures (a parent-child interaction task and the Colorado Childhood Temperament Inventory (CCTI), see below. Six participants were excluded from the parent-child interaction task for not complying with task instructions $(n=3)$, not speaking in English $(n=1)$, or not agreeing to video recording $(n=2))$. Children were between the ages of 4 and $8(M=6.22, S D=$ $1.44)$.

The racial and ethnic makeup of the sample was as follows: $62 \%$ Black, $26 \%$ Hispanic/Latino, 21\% Multiracial, 15\% White, and 3\% Other. Average parental education ranged from 12 to 19 years $(M=13.65, S D=2.31)$. For a subset of participants, parent report of annual family income was available (range: $\$ 2.5 \mathrm{~K}$ to $\$ 175 \mathrm{~K}, M=\$ 45 \mathrm{~K}, S D=\$ 47 \mathrm{~K}$ ).

Child Persistence. Child persistence was measured via parent report on a slightly modified "attention span-persistence" subscale of the CCTI (Rowe \& Plomin, 1977; Cronbach's alpha $=.79$; see Supplemental Material). This measure captures children's global persistence (e.g., "child persists at task until successful") and has been linked to later academic outcomes (McClelland et al., 2014). 
Child Fluid IQ. Children less than 7 years 8 months of age completed the Wechsler Preschool \& Primary Scale of Intelligence (WPPSI) matrix reasoning subscale $(n=25)$. Older children completed the Weschler Intelligence Scale for Children (WISC) matrix reasoning subscale $(n=9)$. Standard scores were used in the analyses.

Parent-Child Interaction Task. Parenting behavior was coded from video of parents and children completing a challenging puzzle task. Parents were given written instructions that said, "See how many of these puzzles your child can make," along with a book of the six hardest Weschler Intelligence Scale (WISC) block design items and one easy demonstration puzzle (see [osf link hidden for blind review] for exact puzzles). Puzzles were taken from the Wechsler Intelligence Scale for Children (WISC) to ensure that children had never seen the puzzles before as they cannot be purchased from a store, and because norms are available, to ensure that the puzzles were sufficiently challenging for children to complete on their own. This paradigm was designed to elicit parents' responses to seeing their children struggle. They were told they would have 5 minutes to complete the task and that a timer would go off when time was up.

Two trained coders independently coded block design task videos (35\% double-coded, interrater reliability: $r=.95-1.0$; Cronbach's alpha $=.99$ across all measures). We coded the number of instances of the following parent behaviors: encouragement (e.g., "keep trying," "you got this"), direct instruction (e.g., "find the red piece," "put it here"), indirect instruction (e.g., parent covers up the picture to focus child on just one block), pedagogical questions (e.g., a question that is intended to teach, such as "do you think your puzzle looks like the picture?"; see Yu, Bonawitz, \& Shafto, 2019), taking over (e.g., parent places a piece of the puzzle for the child), and giving up (e.g., parent encourages quitting puzzle before completion, "let's stop and just try the next one"; for full coding procedure see SM). 
The coding scheme was developed based on video from eight pilot participants for whom CCTI scores were not available. None of the parents used either person or process praise, so praise was not coded in the sample here. All parents used encouragement, direct instruction, indirect instruction, and taking over. Six out of eight parents used pedagogical questions, and one parent gave up. Descriptive statistics of all parenting behaviors are shown in Figure 1.

Analysis plan. This study was exploratory. We tested for correlations between children's persistence and parenting behaviors and for correlations among parenting behaviors. All parenting behaviors, except for direct instruction and encouragement, were not normally distributed (Shapiro-Wilk $p$ s $<.05$ ), so we conducted Spearman correlations (see Figure 1). To correct for multiple comparisons, we used the Benjamini-Yekutieli false discovery rate (FDR; Yekutieli \& Benjamini, 1999).

\section{Results}

As shown in Figure 1, the only parenting behavior that related to children's trait persistence was taking over $(\rho(32)=-.58$, FDR-corrected $p=.004)$. One parent took over at an outlying rate (more than 1.5 times the interquartile range above the third quartile); when this child was removed from the analysis, the relationship was stronger $(\rho(31)=-.67$, FDR-corrected $p<.001)$. Neither child persistence nor taking over was significantly related to age, parent education, income, sex, race, ethnicity, or children's fluid IQ (see SM for details). The relationship between children's trait persistence and taking over was significant after controlling for age, parent education, income, sex, race, ethnicity, and children's fluid IQ $(\rho(32)=-.44, p$ $=.02)$.

Taking over was negatively related to indirect instruction $(\rho(32)=-.53$, FDR-corrected $p$ $=.02$ ), suggesting that these two behaviors trade off when children struggle. Although effect 
sizes were in line with previous work (Prendergast \& MacPhee, 2018), positive parenting behaviors (encouragement, pedagogical questions, and indirect instruction) were not reliably related to child persistence in this small sample.

\section{Discussion}

The results from Study 1 show that parents who took over more often rated their children as less persistent. However, the correlation between parental taking over and child persistence could emerge for many reasons. Parents and children may share personality traits, including impatience or high novelty preference, due in part to shared genetics or experiences (i.e., with stress or trauma) (Deater-Deckard et al., 2006; Sour et al., 2017). Alternatively, parents might take over when they believe their children are genuinely not very persistent. Children might also infer that if their parents usually step in to solve hard problems for them, then they don't need to try. In order to inform parenting interventions, it is important to understand the direction of the relationship between adult taking over and children's persistence. Thus, we next wanted to evaluate whether taking over causes children to be less persistent.

\section{Study 2}

In a preregistered experiment ([osf link hidden for blind review]) we tested whether having an adult take over during a difficult task caused children to be less persistent on a subsequent task. Children were randomly assigned to one of three conditions: a taking over condition, in which the experimenter stepped in and solved hard puzzles for children, a teaching condition, in which the experimenter helped scaffold children's puzzle solving, as well as a no manipulation baseline condition. The teaching condition was inspired by previous studies on supportive parenting (e.g., Frodi et al., 1985; Kelley et al., 2000; Prendergast \& MacPhee, 2018). 


\section{Methods}

Participants. Children were recruited from an urban children's museum. A power analysis indicated that 30 participants per condition were required for a power of .80 (see SM for details). We set an age range of 4- to 5-year-olds to maximize recruitment at the children's museum, which is designed for young children. Importantly, the relationship between parental taking over and children's persistence in Study 1 did not differ between 4- to 5-year-olds and 6to 8 -year-olds $(t(29)=-1.17, p=.25)$. Ninety-six children were recruited for the study, but six were excluded (final $N=90 ; M=4.90$ years, $S D=0.57 ; 47$ female) due to preregistered exclusion criteria (see SM for details). Children were randomly assigned to one of three conditions: taking over, teaching, or baseline $(n=30$ condition $)$. Age $(F(2,87)=0.35, p=.70$, $\left.\eta^{2}=.008\right)$, parental education $\left(F(2,86)=.62, p=.54, \eta^{2}=.01\right)$, and CCTI attention spanpersistence $\left(F(2,82)=0.63, p=.54 \eta^{2}=.02\right)$ did not differ across conditions. The racial and ethnic makeup of the final sample was as follows: 72\% White, 11\% Asian, $9 \%$ Black, 6\% Other, 2\% Hispanic/Latino, and 1\% Multiracial. Parental education ranged from 12 to 20 years $(M=$ $16.34, S D=2.28)$

Procedure. Children were tested individually in a quiet room while their parents completed questionnaires nearby using a tablet. As in Study 1, puzzles were taken from WISC block design (one easy practice item and two challenging items; see [osf link hidden for blind review] for puzzles). In both the teaching and taking over conditions, the experimenter demonstrated how to make the practice picture and then told the child it was her turn to make the pictures (see [osf link hidden for blind review] for script).

In the taking over condition, the experimenter let the child try to make the puzzle for approximately 10 seconds, then "took over" by saying "Hmm...this is hard, why don't I just do it 
for you" and placed all the blocks correctly in the puzzle. The experimenter repeated this procedure again with a second puzzle. In the teaching condition, children worked sequentially on the same two puzzles, but this time the experimenter helped scaffold the child's puzzle building with indirect instruction (e.g. covering up half of the puzzle with her hands), direct instruction (e.g. "find a piece that is half white and half red"), and pedagogical questions (e.g. "does that look like the picture?"), ensuring that the child completed the puzzle without the experimenter physically placing any puzzle pieces. In both conditions, the experimenter reacted positively on completion of the puzzles.

Next, the experimenter took away the puzzle stimuli and introduced the child to another toy. The baseline condition did not have a pre-puzzle phase in order to see how children interacted with the persistence toy with no prior information (just as in Leonard, Garcia, \& Schulz, 2019). The experimenter took out a wooden box toy and shook it, saying, "This is your toy! It sounds like something's inside of there. I wonder if you can open it up? Why don't you play with it and see if you can open it up? If at any point you feel like you can't do it, you can play with this other toy [a water toy] and I'll come help you." The experimenter then gave the child a hollow wooden box toy with an eraser inside. The box looked like it could open a few ways, but in actuality could only be opened with a secret notch that was glued shut. We chose the wooden box task for our dependent measure because it was novel, not clearly within or outside of children's ability range, and unlikely to be related to IQ since it is unsolvable and offers no affordances to track progress. The experimenter placed the water toy about $30 \mathrm{~cm}(M=33 \mathrm{~cm}$, $S D=6 \mathrm{~cm}$ ) from the child and moved out of the child's line of sight while she "reviewed paperwork." The experiment ended when the child started playing with the water toy or after four minutes, whichever came first (similar in design to Leonard et al., 2019; see Figure 2). 
Analysis plan. Child persistence was operationalized as total time spent trying to open the box. Persistence was coded from video by two coders, one blind to condition $(100 \%$ of videos were double-scored with inter-rater reliability $r>.99)$. Children's persistence did not adhere to a normal distribution (Shapiro-Wilk $p<.001$, Skewness $=.9$ ), so we ran nonparametric Wilcoxon rank-sum tests to compare conditions. We preregistered first comparing the taking over condition to the teaching condition and then, to understand directionality, running follow-up tests comparing each condition to baseline.

\section{Results}

Children in the taking over condition did not persist as long as children in the teaching condition ( $W=237.5, p=.002, r=-.41$; see Figure 3, Table 1 for means and medians), or as long as children in the baseline condition $(W=606.5, p=.02, r=-.30)$. Children's persistence did not significantly differ between the teaching and baseline conditions $(W=393, p=.40, r=$ $-.11)$.

A number of control analyses were conducted. No differences were found by condition for the distance the water toy was placed from the child $\left(F(2,87)=0.60, p=.55, \eta^{2}=.01\right.$; means per condition - taking over: $32.8 \mathrm{~cm}$, teaching: $32.9 \mathrm{~cm}$, baseline: $34.4 \mathrm{~cm}$ ) or the number of times the experimenter talked to the child during the persistence part of the task $(F(2,87)=0.25, p$ $=.78, \eta^{2}=.01$; means per condition - taking over: 0.97 , teaching: 1.23 , baseline: 1.03$)$. Additionally, because the experimenter might have conveyed more positive affect while handing the toy to the child in the teaching and baseline conditions, a second coder rated the experimenter's positive affect while handing over the toy on a Likert scale (1(negative) 7 (positive)), blind to conditions. Experimenter affect did not differ by condition $(F(2,87)=0.19$, $p=.83, \eta^{2}=.004$; means per condition - taking over: 4.33 , teaching: 4.43 , baseline: 4.37 ). To 
further make sure that experimenter behavior did not differ by condition during the introduction of the second toy, the same coder (blind to condition) tried to guess what condition (taking over, teaching, no idea) each child was in just from watching the introduction of the wooden box toy for the taking over and teaching conditions. They guessed $25 \%$ percent correctly, not significantly different from chance $\left(\chi^{2}(1, n=60)=0.33, p=.85\right)$. Thus, experimenter behavior didn't systematically vary by condition during the persistence part of the task.

In preregistered secondary analyses, parent ratings of persistence (CCTI) did not relate to children's persistence on the box task (all $p \mathrm{~s}>.2$; see SM for details). Across conditions, parent education was negatively related to children's persistence on the box task $(\rho(88)=-.25, p=.02)$. There was a significant negative relationship between parent education and persistence in the baseline condition $(\rho(28)=-.47, p=.009)$ and in the taking over condition $(\rho(28)=-.44, p$ $=.02)$, but not in the teaching condition $(\rho(28)=.02, p=.90)$. Within and across conditions, children's persistence did not relate to age, race, or ethnicity (see SM for details). However, across conditions, but not within condition, girls persisted more than boys $(W=1274.5, p=.03, r$ $=-.22)$.

\section{Discussion}

Study 2 shows that taking over impairs persistence on a subsequent task. However, taking over is common, especially when a task needs to be done quickly or requires adult skills. This raises the question of what adults can do to reframe taking over so that it has less of an impact on children's motivation. In Study 3, we explored one potential way adults can reframe taking over and tested whether it reversed the demotivating effects.

\section{Study 3}


In a second preregistered experiment ([osf link hidden for blind review]), we tested whether adults can take over without demotivating children by reframing the context to be one in which the experimenter took over because it was their turn in a turn taking game. We compared children's persistence in the taking over condition to the turns condition, in which the experimenters' actions were the same, but their words differed. We hypothesized that children in the turns condition would not infer that the experimenter would always take over when tasks were hard, but rather that they took over in the game because they were adhering to the norms of a "turn taking game". Thus, we predicted that children in the turns condition would try harder than children in the taking over condition.

\section{Methods}

Participants. Children were recruited from an urban children's museum. A power analysis indicated that 30 participants per condition were required for a power of .80 (see SM for details). Seventy children were recruited for the study, but ten were excluded (final $N=60 ; M=$ 4.79 years, $S D=0.49 ; 26$ female) due to preregistered exclusion criteria (see SM for details). Children were randomly assigned to one of two conditions: taking over or turns $(n=$ $30 /$ condition $).$ Age $(t(58)=-1.19, p=.24, d=.31)$ and parental education $(t(58)=0.21, p=.83$ $d=.05)$ did not differ across conditions. The racial and ethnic makeup of the final sample was as follows: $42 \%$ White, 25\% Black, 12\% Asian, 8\% Other, 10\% Multiracial, and 17\% Hispanic/Latino. Parental education ranged from 12 to 20 years $(M=16.53, S D=2.44)$.

The same stimuli from Study 2 were used here except for one small modification. The first block design given to children was switched to a slightly harder one because one child in 
Study 2 was able to solve it before the experimenter took over (see [osf link hidden for blind review] for exact materials).

Procedure. As in Study 2, in both conditions, the experimenter demonstrated how to make the practice picture and then told the child it was their turn to make the pictures (see [osf link hidden for blind review] for exact script). The taking over condition was the same as in Study 2 .

In the turns condition, the experimenter explained that they were going to play a game in which they took turns. The experimenter actions were the same as those in the taking over condition - they let the child try to make the puzzle for around 10 seconds and then took overhowever, when they took over they said "now it's my turn." Importantly, they also noted that the puzzles were hard in the turns condition, saying "hmm this is hard." After the experimenter finished the puzzle, they introduced children to the second puzzle saying, "your turn," and then took over after 10 seconds to complete the second puzzle. Just as in Study 2, the experimenter took away the puzzles and introduced the child to the impossible wooden box. See methods in Study 2 for specifics on how persistence was measured (see Figure 2).

Analysis plan. Child persistence was again operationalized as total time spent trying to open the box. Persistence was coded from video by two coders, one blind to condition (100\% of videos were double-scored with inter-rater reliability $r>.99$ ). Again, we expected (see osf prereg link) and confirmed that children's persistence did not adhere to a normal distribution (Shapiro-Wilk $p<.001$, Skewness $=1.15)$, so we ran non-parametric Wilcoxon rank-sum tests to compare conditions. We preregistered comparing the taking over condition to the turns condition.

\section{Results}


Children in the turns condition persisted longer on the box task than children in the taking over condition, although not significantly $(W=340, p=.10, r=-.21$; see Figure 4 , Table 1 for means and medians).

Given the trend level result, we ran a simulated power analysis to determine the sample that would be needed to find a significant result with power $=.80$. Using the data from this experiment, we ran 10,000 Wilcoxon rank-sum tests with various samples bootstrapped with replacement and coded each significant model as 1 and each insignificant model as 0 . Dividing the number of significant models by the total number of simulations gives us an estimate of our simulated studies' power. We found that we would need 95 participants per condition for a power of .80 .

Within and across conditions, children's persistence did not relate to parent education, gender, race, or ethnicity (see SM for details). Although there was no significant relationship between age and children's persistence across conditions $(\rho(58)=.15, p=.26)$, or in the turns condition $(\rho(28)=-.13, p=.49)$, there was a significant positive relationship between age and children's persistence in the taking over condition $(\rho(28)=.41, p=.02)$.

\section{Discussion}

We found that children in the turns condition persisted longer than children in the taking over condition, although not significantly. Thus, changing the context and language of the adult who took over was not enough to reverse the demotivating effect of an adult solving a task for a child.

\section{General Discussion}


We found that when adults take over and solve hard problems for children, children persist less. First, we showed that parental taking over negatively relates to parent report of child persistence. Then, in an experimental study, we demonstrated that taking over causes children to persist less on a subsequent difficult task. Finally, in a second experimental study, we found that changing the language and context of taking over slightly, but not significantly, ameliorated the negative impact of taking over on children's persistence.

The current investigation adds to the decades-long research interest in the relationship between parenting practices and children's persistence. The majority of studies have been on behaviors that improve persistence, with experimental work pointing to process praise (which is rare in real life), and observational work pointing towards parental warmth, scaffolding, and encouragement. Our findings suggest that it is easier to demotivate children than to motivate them: effect sizes for taking over and giving up on children's persistence were larger than effect sizes for all positive behaviors, and taking over had a larger impact than supportive teaching as compared to baseline.

Although there has been prior work on negative impacts on children's persistence, it focused on the construct of "intrusive parenting" (Mokrova et al., 2012), which includes not only the taking over behavior studied here, but also behaviors such as providing unsolicited help and instructions, which would fall into the "direct instruction" code in this study (Grolnick et al., 2002; Mokrova et al., 2012). By studying specific behaviors, rather than constructs that include multiple behaviors, our approach points towards more specific intervention strategies to improve children's persistence. Furthermore, we show that taking over has a negative impact on state persistence even when the agent is a new acquaintance, suggesting that interventions could be targeted broadly towards people who work with children. 
Taking over decreases children's persistence through at least two possible cognitive mechanisms. One, taking over signals to children that these tasks are too hard for them and that they are unlikely to be able to succeed on their own (see Gweon \& Schulz, 2011, for work showing that children distinguish whether tasks are difficult due to the agent's ability or the task not working). Two, taking over teaches children that when tasks are hard, an adult will do them for you. Note that children in the teaching condition may also learn that they are unlikely to be able to succeed on their own, since the adult helps them solve the puzzles. The fact that children in the teaching condition persist more than those in the taking over condition indicates that thinking the task requires adult help is not sufficient to demotivate children - they must also believe either that they are incapable of the task or that the adult will likely step in to do the task for them. Experimental work could illuminate which inferences most strongly demotivate children.

In Study 1, the relationship between encouragement, pedagogical questions, and indirect instruction and children's persistence was positive but did not reach significance, possibly due to the relatively small sample size. Similarly, children in the teaching condition of Study 2 did not persist more than children in the baseline condition. Although children in the teaching condition did successfully solve the puzzles with help from the experimenter, the puzzles were difficult, and children did not have any evidence that the box toy was actually possible to open. The combined expectations of difficulty and uncertainty of success have been shown to negatively impact children's persistence compared to conditions in which children know the task is difficult, but possible (Leonard et al., 2019). Teaching may bolster children's persistence in contexts in which children know that the probability of their success is higher. 
Children in the teaching condition had more time to interact with the puzzles than children in the taking over condition. Thus, one alternative explanation is that children in the teaching condition learned some sort of broader social norm that they should persist on the tasks at hand. However, we think this interpretation is unlikely considering that children in the teaching condition did not persist more than children in the baseline condition who did not previously interact with the puzzles or an experimenter.

We were not able to reverse the demotivating effects of taking over by reframing these actions in the context of a game in which the experimenter and child take turns. In line with previous work, our results suggest that, at least in the current context, actions speak louder than words (Leonard, Garcia, \& Schulz, 2019; Rushton, 1975). Importantly, we did replicate the taking over result in Study 2 - the means and medians across studies are nearly identical (see Table 1). A simulated power analysis revealed that the turns manipulation may just be a weak effect that would appear only with a large sample. Future work may try to increase the salience of the context change, perhaps by having the adult and child take more turns per puzzle (instead of the adult just completing it in one go). Furthermore, different contexts (parents being in a rush vs. a dangerous situation) may require different strategies to ameliorate the negative effects of taking over.

One open question is what causes parents to take over. Parents who took over more often gave less direct instruction, and also tended to give up more. Perhaps parents who are stressed, easily frustrated, or impatient take over more, and have less bandwidth to patiently teach (Deater-Deckard, 1998). Perhaps parents learn to take over from observing their own parents or other models in their communities (Neppl, Conger, Scaramella, \& Ontai, 2009), or have children 
who elicit more help. Importantly, taking over was not related to socioeconomic status, race, or ethnicity.

Parent education and income were not related to parent report of persistence, nor to taking over. However, children whose parent had completed less formal education persisted longer on the box task in Study 2, but not in Study 3. It is therefore unclear whether persistence is indeed stronger in children from lower SES backgrounds (in contrast to findings from Brown, 2009 \& Evans, 2014, but consistent with a "hidden talents in harsh conditions" framework, see Ellis, Bianchi, Griskevicius \& Frankenhuis, 2017), or whether children from different backgrounds interpret the specific instructions and features of the task differently. For example, children from diverse backgrounds may have different familiarity with these objects (even though we attempted to pick novel objects), differently assess the value of completing the goal (opening the box) or discovering new information (what's inside the box), or have different expectations of how to behave in the experimental context. It is difficult to interpret individual differences in the context of an experiment with randomized assignment because children's backgrounds may interact with condition assignment. For example, cultural factors may change the way adult taking over impacts children's persistence. In some cultures, children are more involved in adult work (Morelli et al., 2003), which may alter the frequency of adult taking over and in turn, children's appraisal of this action. Thus, children from cultural backgrounds who are more accustomed to adult taking over may not be as negatively impacted by it.

We used different measures of persistence to capture trait persistence (parent report) and state persistence (time working on an unsolvable puzzle). These two measures did not significantly relate to each other in Study 2 . The parent report measure necessarily measures persistence from the parent's perspective, and children likely vary in behavior around different 
adults (i.e., persist more around adults who take over less). Further, parents likely vary in their ability to recognize and extract patterns in their children's behavior. The box task, on the other hand, has only been previously used in experimental manipulations and thus, has not yet been validated as an individual difference measure. In other words, children's performance on the box task may reflect their persistence in a given moment, but not their average, or trait, persistent behavior across days. The parent report measure (CCTI attention-span persistence), on the other hand, likely captures children's average persistence across days better than their persistence on any given day. However, future work is needed to develop naturalistic, reliable, and repeatable measures of children's persistence and to explore how taking over impacts persistence across longer time scales.

This study had three main limitations. First, we observed parenting behavior during a semi-structured in-lab task, which may not reflect broader parenting behaviors outside of the lab. Second, although Study 2 showed that the experience of taking over can cause children to persist less, it does not prove that relationships observed in Study 1 were environmental, as they could be due in part to shared genetics (Deater-Deckard et al., 2006). Furthermore, demographics differed between studies and neither matched the demographics of the United States (though note that this is a common problem in psychology research). Third, we did not collect data on children's learning, so we could not test whether parenting or persistence measures relate to academic outcomes or other important real-world behaviors.

In sum, we found that children persist less when adults take over. Our results suggest that interventions to help parents and educators take over less, and let children struggle in some situations, may lead to greater persistence in young children. Future work is needed to determine the best way to encourage parents to change their behaviors, and to test whether behavior change 
enhances persistence over longer time scales. That said, there are clearly situations in which adults need to take over, both under ordinary time pressures, and in urgent or high-risk situations. A critical question moving forward is how adults can take over without demotivating children. Our effort to undo the negative impact of taking over by reframing it as "taking turns" was not successful. Perhaps reassuring children that they are capable, and that they do not need (and will not get) help, might be more impactful. However, considering that in many situations, children pay more attention to adults' actions than to their words (Leonard et al., 2019; Rushton, 1975), verbally reframing taking over might not be enough. Luckily, many parenting interactions are not especially urgent: when possible, it might be worth leaving the house ten minutes later to let your child work hard to tie her own shoes. 


\section{References}

Banerjee, P. N., \& Tamis-LeMonda, C. S. (2007). Infants' persistence and mothers' teaching as predictors of toddlers' cognitive development. Infant Behavior and Development, 30(3), 479-491. doi:10.1016/j.infbeh.2006.12.001

Brown, E. D. (2009). Persistence in the face of academic challenge for economically disadvantaged children. Journal of Early Childhood Research, 7(2), 173-184. doi:10.1177/1476718X09102650

Cimpian, A., Arce, H.M. C., Markman, E. M., \& Dweck, C. S. (2007). Subtle linguistic cues affect children's motivation. Psychological Science, 18(4), 314-316. doi:10.1111/j.14679280.2007.01896.x

Cutcher-Gershenfeld, J. (1991). The impact on economic performance of a transformation in workplace relations. ILR Review, 44(2), 241-260.doi: 10.1177/001979399104400204

Deater-Deckard, K. (1998). Parenting stress and child adjustment: Some old hypotheses and new questions. Clinical Psychology: Science and Practice, 5(3), 314-332. doi:10.1111/j.14682850.1998.tb00152.x

Deater-Deckard, K., Petrill, S. A., Thompson, L. A., \& DeThorne, L. S. (2006). A longitudinal behavioral genetic analysis of task persistence. Developmental Science, 9(5), 498-504. doi:10.1111/j.1467-7687.2006.00517.x

Eskreis-Winkler, L., Duckworth, A. L., Shulman, E. P., \& Beal, S. (2014). The grit effect: Predicting retention in the military, the workplace, school and marriage. Frontiers in Psychology, 5, 36. doi: 10.3389/fpsyg.2014.00036

Evans, G. W. (2016). Childhood poverty and adult psychological well-being. Proceedings of the National Academy of Sciences, 113(52), 14949-14952. doi:10.1073/pnas.1604756114 
Ellis, B. J., Bianchi, J., Griskevicius, V., \& Frankenhuis, W. E. (2017). Beyond risk and protective factors: An adaptation-based approach to resilience. Perspectives on Psychological Science, 12(4), 561-587. doi:10.1177/1745691617693054

Eskreis-Winkler, L., Shulman, E. P., Beal, S. A., \& Duckworth, A. L. (2014). The grit effect: Predicting retention in the military, the workplace, school and marriage. Frontiers in Psychology, 5(36), 1-12. doi:10.3389/ fpsyg.2014.00036

Frodi, A., Bridges, L., \& Grolnick, W. (1985). Correlates of mastery-related behavior: A shortterm longitudinal study of infants in their second year. Child Development, 56(5), 12911298.

Gottfried, A. E. (1985). Academic intrinsic motivation in elementary and junior high school students. Journal of Educational Psychology, 77(6), 631-645. doi:10.1037/00220663.77.6.631

Gunderson, E. A., Gripshover, S. J., Romero, C., Dweck, C. S., Goldin-Meadow, S., \& Levine, S. C. (2013). Parent praise to 1- to 3-year-olds predicts children's motivational frameworks 5 years later. Child Development, 84(5), 1526-1541. doi:10.1111/cdev.12064

Grolnick, W. S., Gurland, S. T., DeCourcey, W., \& Jacob, K. (2002). Antecedents and consequences of mothers' autonomy support: An experimental investigation. Developmental Psychology, 38(1), 143-155. doi:10.1037/0012-1649.38.1.143

Groves, T., Hong, Y., McMillan, J., \& Naughton, B. (1994). Autonomy and incentives in Chinese state enterprises. The Quarterly Journal of Economics, 109(1), 183-209. doi: $10.2307 / 2118432$ 
Gweon, H., \& Schulz, L. (2011). 16-month-olds rationally infer causes of failed actions. Science, 332(6037), 1524. doi:10.1126/science. 1204493

Ichniowski, C., Shaw, K., \& Prennushi, G. (1995). The effects of human resource management practices on productivity (No. w5333). National Bureau of Economic Research. doi: $10.3386 /$ w5 5333

Kamins, M. L., \& Dweck, C. S. (1999). Person versus process praise and criticism: Implications for contingent self-worth and coping. Developmental Psychology, 35(3), 835-847. doi:10.1037/0012-1649.35.3.835

Kelley, S. A., Brownell, C. A., \& Campbell, S. B. (2000). Mastery motivation and selfevaluative affect in toddlers: Longitudinal relations with maternal behavior. Child Development, 71(4), 1061-1071. doi:10.1111/1467-8624.00209

Leonard, J. A., Garcia, A., \& Schulz, L. E. (2019). How adults' actions, outcomes, and testimony affect preschoolers' persistence. Child Development. Advance online publication. doi:10.1111/cdev.13305

Leonard, J. A., Lee, Y., \& Schulz, L. E. (2017). Infants make more attempts to achieve a goal when they see adults persist. Science, 357(6357), 1290-1294. doi:10.1126/science.aan2317

Lucca, K., Horton, R., \& Sommerville, J. A. (2019). Keep trying!: Parental language predicts infants' persistence. Cognition, 193, doi:10.1016/j.cognition.2019.104025

Lucca, K., \& Sommerville, J. A. (2018). The little engine that can: Infants' persistence matters. Trends in Cognitive Sciences, 22(11), 965-968. doi:10.1016/j.tics.2018.07.012 
Martin, A., Ryan, R. M., \& Brooks-Gunn, J. (2013). Longitudinal associations among interest, persistence, supportive parenting, and achievement in early childhood. Early Childhood Research Quarterly, 28(4), 658-667. doi:10.1016/j.ecresq.2013.05.003

McClelland, M. M., Acock, A. C., Piccinin, A., Rhea, S. A., \& Stallings, M. C. (2013). Relations between preschool attention span-persistence and age 25 educational outcomes. Early Childhood Research Quarterly, 28(2), 314-324. doi:10.1016/j.ecresq.2012.07.008

Messer, D. J., McCarthy, M. E., McQuiston, S., MacTurk, R. H., Yarrow, L. J., \& Vietze, P. M. (1986). Relation between mastery behavior in infancy and competence in early childhood. Developmental Psychology, 22(3), 366-372. doi:10.1037/00121649.22.3.366

Mischel, W., Shoda, Y., \& Rodriguez, M. I. (1989). Delay of gratification in children. Science, 244(4907), 933-938. doi:10.1126/science.2658056

Mokrova, I. L., O’Brien, M., Calkins, S. D., Leerkes, E. M., \& Marcovitch, S. (2013). The role of persistence at preschool age in academic skills at kindergarten. European Journal of Psychology of Education, 28(4), 1495-1503. doi:10.1007/s10212-013-0177-2

Mokrova, I. L., O'Brien, M., Calkins, S. D., Leerkes, E. M., \& Marcovitch, S. (2012). Links between family social status and preschoolers' persistence: The role of maternal values and quality of parenting. Infant and Child Development, 21(6), 617-633. doi:10.1002/icd.1761

Morelli, G. A., Rogoff, B., \& Angelillo, C. (2003). Cultural variation in young children's access to work or involvement in specialised child-focused activities. International Journal of Behavioral Development, 27(3), 264-274. doi: 10.1080/01650250244000335 
Mueller, C. M., \& Dweck, C. S. (1998). Praise for intelligence can undermine children's motivation and performance. Journal of Personality and Social Psychology, 75(1), 33 52. doi:10.1037/0022-3514.75.1.33

Neppl, T. K., Conger, R. D., Scaramella, L. V., \& Ontai, L. L. (2009). Intergenerational continuity in parenting behavior: Mediating pathways and child effects. Developmental Psychology, 45(5), 1241. doi: 10.1037/a0014850

Prendergast, S., \& MacPhee, D. (2018). Parental contributors to children's persistence and school readiness. Early Childhood Research Quarterly, 45(4), 31-44. doi:10.1016/j.ecresq.2018.05.005

Reeve, J., Jang, H., Carrell, D., Jeon, S., \& Barch, J. (2004). Enhancing students' engagement by increasing teachers' autonomy support. Motivation and Emotion, 28(2), 147-169. doi: 10.1023/B:MOEM.0000032312.95499.6f

Rowe, D. C., \& Plomin, R. (1977). Temperament in early childhood. Journal of Personality Assessment, 41(2), 150-156. doi:10.1207/s15327752jpa4102_5

Rushton, J. P. (1975). Generosity in children: Immediate and long-term effects of modeling, preaching, and moral judgment. Journal of Personality and Social Psychology, 31(3), 459-466. doi:10.1037/h0076466

Suor, J. H., Sturge-Apple, M. L., Davies, P. T., \& Cicchetti, D. (2017). A life history approach to delineating how harsh environments and hawk temperament traits differentially shape children's problem-solving skills. Journal of Child Psychology and Psychiatry, 58(8), 902-909. doi: 10.1111/jcpp.12718

Walker, J. M. (2008). Looking at teacher practices through the lens of parenting style. The Journal of Experimental Education, 76(2), 218-240. doi: 10.3200/JEXE.76.2.218-240 
Yarrow, L. J., Morgan, G. A., Jennings, K. D., Harmon, R. J., \& Gaiter, J. L. (1982). Infants' persistence at tasks: Relationships to cognitive functioning and early experience. Infant Behavior and Development, 5(2-4), 131-141. doi:10.1016/S0163-6383(82)80023-4

Yekutieli, D., \& Benjamini, Y. (1999). Resampling-based false discovery rate controlling multiple test procedures for correlated test statistics. Journal of Statistical Planning and Inference, 82(1-2), 171-196. doi:10.1016/S0378-3758(99)00041-5

Yu, Y., Bonawitz, E., \& Shafto, P. (2019). Pedagogical questions in parent-child conversations. Child Development, 90(1), 147-161. doi:10.1111/cdev.12850

Zimmerman, B. J., \& Blotner, R. (1979). Effects of model persistence and success on children's problem solving. Journal of Educational Psychology, 71(4), 508-513. doi:10.1037/00220663.71.4.508 0/0/00 0:00:00 AM 


\section{Figures}
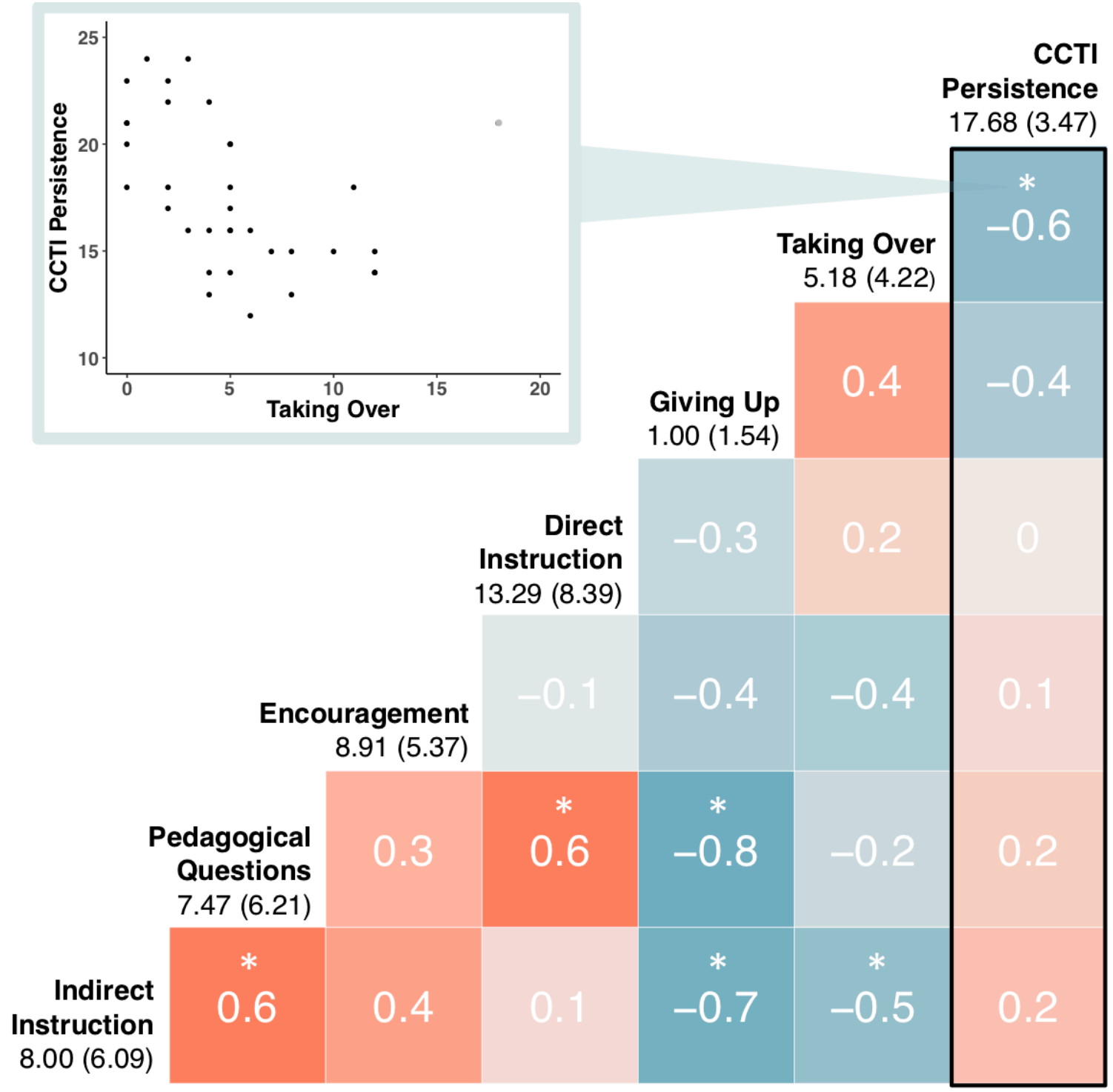

Figure 1. Spearman correlations between parenting behaviors and CCTI attention span-persistence in Study 1. Means and standard deviations are reported for all parenting behaviors as mean $(S D)$ below their corresponding labels. Correction for multiple comparisons was conducted with FDR. * corrected $p$ $<.05$. The only parenting behavior that significantly related to CCTI attention span-persistence with FDR correction was taking over. 


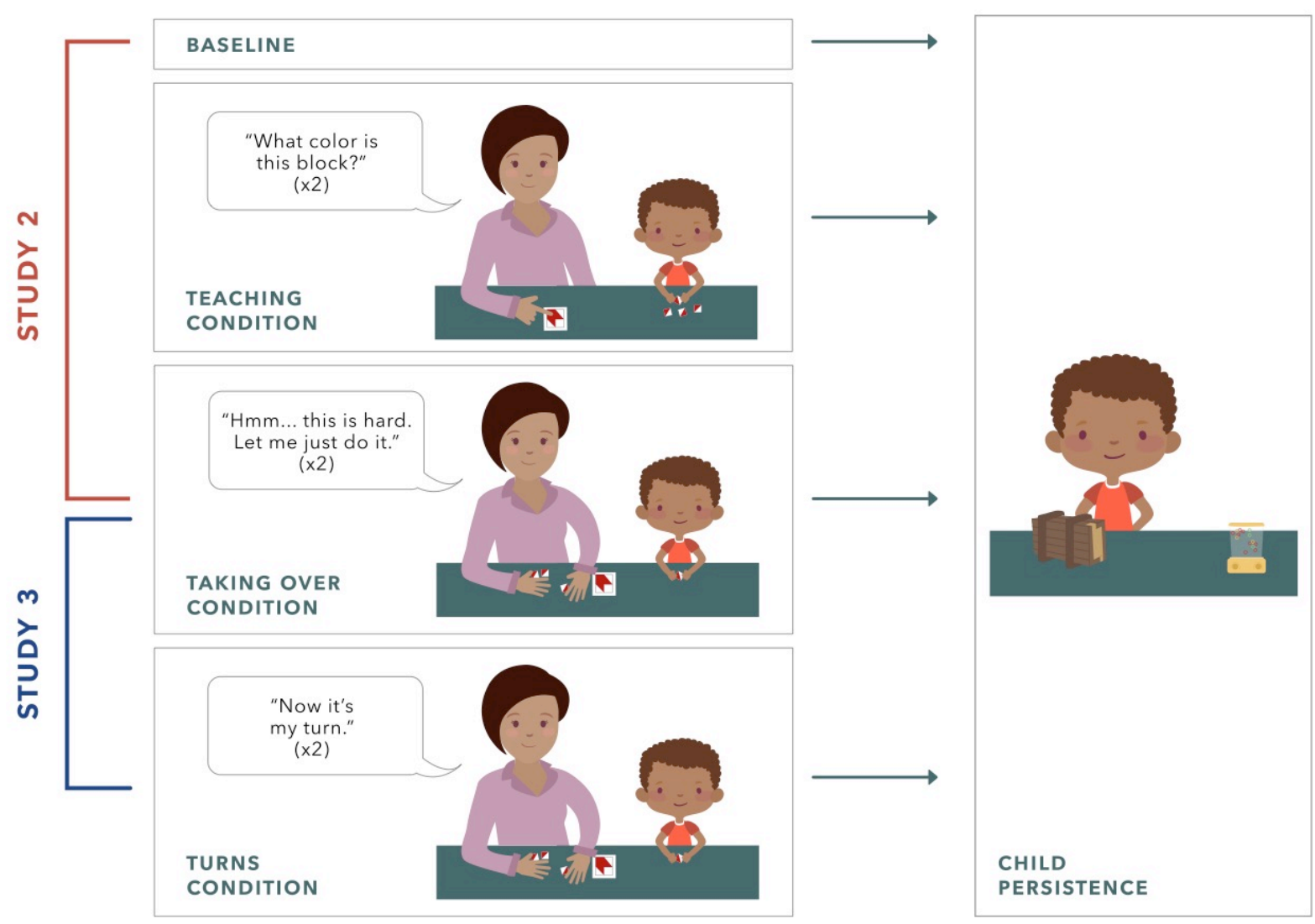

Figure 2. Schematic of Study 2 and 3. In Study 2, children were randomly assigned to one of three conditions: taking over, teaching, or baseline. In the taking over condition, the experimenter stepped in and solved two puzzles for children. In the teaching condition, the experimenter helped children solve two puzzles. In the baseline condition, there was no demonstration. In Study 3, children were randomly assigned to the taking over condition (same setup as in Study 2), or the turns condition, in which the experimenter also took over and solved puzzles, but in the context of a game in which they take turns. In both studies, children were given a wooden box to play with after the manipulation. The box sounded like it had something inside of it but was secretly impossible to open. Children were told that they could play with the water toy when they were all done playing with the box and were timed out at four minutes. 


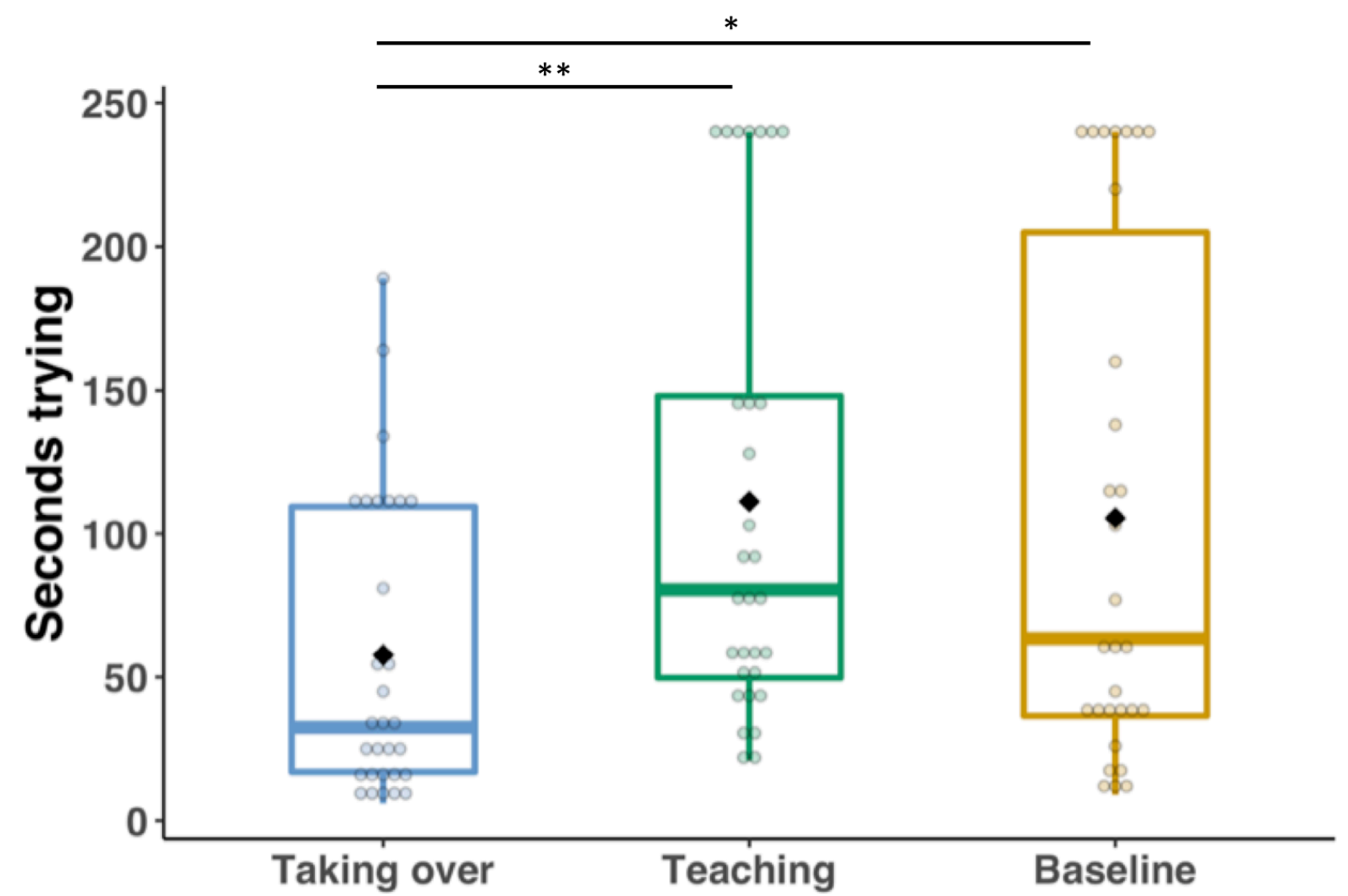

Figure 3. Results from Study 2. Children persisted significantly longer in the teaching condition and in the baseline condition than in the taking over condition. Black diamonds represent group means. ${ }^{*} p<.05$, $* * p<.01$. 


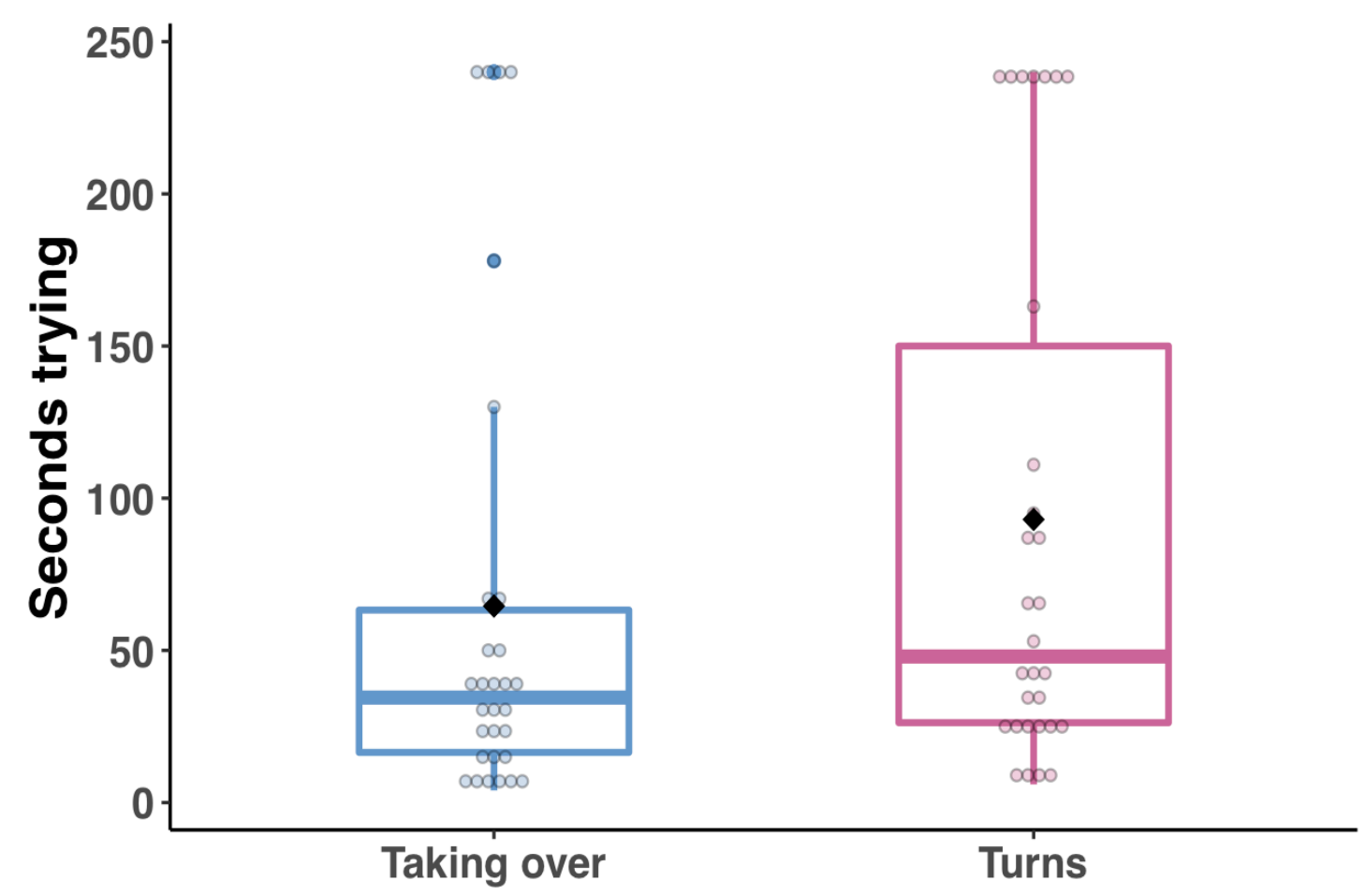

Figure 4. Results from Study 3. There was no statistical difference in children's persistence between the taking over and turns conditions. Black diamonds represent group means. 
Table 1. Medians, and means and their 95\% confidence intervals from a bootstrap with 10,000 samples for child's persistence (sec) in Study 2 and Study 3.

\begin{tabular}{lllll}
\hline & & Median & Mean & Mean 95\% CI \\
\hline Study 2 & Taking Over & 32.5 & 57.7 & $38.5,75.0$ \\
& Teaching & 80.5 & 111.0 & $82.4,138.2$ \\
& Baseline & 63.5 & 106.0 & $73.2,135.8$ \\
\hline Study 3 & Taking Over & 34.5 & 64.6 & $34.93,90.00$ \\
& Turns & 48.0 & 93.0 & $61.40,122.37$ \\
\hline
\end{tabular}




\section{Supplementary Materials \\ Children Persist Less When Adults Take Over}

\section{Study 1}

\section{Methods}

CCTI attention span-persistence. The following questions, slightly modified from Rowe \& Plomin (1977), made up the CCTI attention span-persistence subscale. All questions were answered from 1 (not like the child at all) to 5 (a lot like the child). Scores from the following questions were summed to create the attention span-persistence subscale. Questions marked with " $\mathrm{R}$ " were reverse scored.

1. Child gives up easily when difficulties are encountered (R)

2. Child persists at a task until successful

3. Child goes from toy to toy quickly $(\mathrm{R})$

4. With a difficult toy or game, child gives up quite easily $(\mathrm{R})$

5. Child plays with a single toy or game for long periods of time

Parent-child interaction task. The block design task occurred after a wordless picture book, and before a box of novel toys. The full parent-child interaction task was modeled after the Three Bags Task. We focused our analyses on the block design task because we were interested in how parents would behave when their child was faced with a challenge. The block design task was the only portion of the parent-child interaction paradigm with explicit, semi-structured goals for the child.

Coding parent behaviors. A master coder trained two coders to high reliability on 8 practice videos using the coding scheme outlined in SM Table S1, discussing discrepancies. One trained coder scored all videos in this analysis. A second trained coder scored $35 \%$ of the videos without discussing coding with the first coder (interrater reliability: $r=.95-1.0$; see SM Figure S1).

\section{Results}

Child persistence was not related to age $(r(32)=.25, p=.15)$, parent education $(r(32)$ $=.30, p=.09)$, income $(r(32)=.08, p=.66)$, fluid IQ $(r(32)=-.01, p=.96)$, sex $(t(32)=-1.17, p$ $=.25, d=.41)$, race $(F(3,30)=1.62, p=.21)$, or ethnicity $(t(32)=0.24, p=.81, d=.09)$. Taking over was also not related to age $(\rho(32)=-.22, p=.22)$, parent education $(\rho(32)=-.26, p=.13)$, income $(\rho(32)=-.08, p=.67)$, fluid IQ $(\rho(32)=.09, p=.61)$, $\operatorname{sex}(W=170, p=.35, r=-.16)$, race $(H(3)=4.64, p=.20)$, or ethnicity $(W=129, p=.53, r=-.11)$.

\section{Study 2}




\section{Methods}

Participants and materials. We ran a pilot experiment in which we randomly assigned 13 children to the "teaching" condition and 15 to the "taking over" condition. To determine our sample size for the current study, we ran a simulation based on data from this pilot because we expected similar effect sizes. We ran 10,000 Wilcoxon rank-sum tests with various samples bootstrapped with replacement from the initial data set. Then we coded each significant model as 1 and each insignificant model as 0 . Dividing the number of significant models by the total number of simulations gives us an estimate of our simulated studies' power. We found that the simulated power was .8 for $\mathrm{n}=30$ subjects/condition.

Children were excluded ( $n=6 ; 1$ per each reason) for the following pre-registered exclusion criteria: no video, experimenter administration error, another child entering the room during testing, another child having seen someone do the study before, solving the puzzle before the experimenter could take over, and taking more than seven minutes to solve a puzzle in the teaching condition.

A booklet of three WISC block design puzzles, including one easy practice item (see [osf link hidden for blind review] for exact puzzles) were used. Additionally, one $18.49 \times 8.51 \times 8.51$ $\mathrm{cm}$ wooden box was used, with a rubber eraser inside to demonstrate that the box was hollow and could be opened. The box looked like it could open in a few different ways, but actually opened through a secret sliding notch that was glued shut. Children indicated when they were all done playing with the box toy by playing with a small handheld water toy $(16.5 \times 10.2 \times 3.8 \mathrm{~cm})$. See Figure 2 for a schematic of the stimuli and procedure.

Procedure details. Children were tested individually in a quiet room in an urban children's museum. The child and the experimenter sat diagonally at the corner of a long table. There was a second chair placed to the right of the child so that they could move to it during the persistence portion of the study to play with the water toy placed in front of it (see Methods).

During the persistence portion of the task, the experiment followed a specific protocol. If the child stopped touching the box toy for $\sim 5$ seconds, but did not move to the water toy, the experimenter asked if they were still playing. If they said "no," the experimenter told them to play with the water toy. If the child said "this is too hard/ I need help/ I'm all done" without moving to the water toy, the experimenter said, "OK if you feel like you can't do it, you can go play with the water toy and I'll come help." The experiment was terminated when the child played with the water toy, or after four minutes, whichever came first (similar in design to Leonard, Garcia, \& Schulz, 2019). The experimenter always ended by saying, "Great job trying! These boxes are hard to open up! Let's work on one together!" The child was given a different box and, working with the experimenter, successfully opened the box in the end.

\section{Results}

Within and across conditions, parent report of persistence (CCTI) did not relate to children's persistence on the box task (across: $\rho(88)=.04, p=.73$, taking over: $\rho(28)=-.18, p$ $=.36$, teaching: $\rho(28)=-.06, p=.78$, baseline: $\rho(28)=.22, p=.25)$. Across conditions, parent education was negatively related to children's persistence on the box task $(\rho(87)=-.25, p=.02)$. There was a significant negative relationship between parent education and persistence in the baseline condition $(\rho(28)=-.47, p=.009)$ and in the taking over condition $(\rho(27)=-.44, p$ $=.02$ ), but not in the teaching condition $(\rho(28)=.02, p=.90)$. Within and across conditions, 
children's persistence did not relate to age (across: $\rho(88)=-.05, p=.65$, taking over: $\rho(28)=$ $-.004 p=.98$, teaching: $\rho(28)=-.11, p=.57$, baseline: $\rho(28)=.04, p=.82)$, race (across: $H(4)=$ 4.16, $p=.38$, taking over: $H(3)=1.34, p=.72$, teaching: $H(3)=2.46, p=.48$, baseline: $H(4)=$ $1.83, p=.77$ ), or ethnicity (across: $W=51, p=.37, r=-.09$, teaching: $W=24, p=.77, r=-.05$, both taking over and baseline only had one ethnicity represented). However, across conditions girls persisted more than boys ( $W=1274.5, p=.03, r=-.22$ ). This effect of sex was not present within conditions (taking over: $W=115.5, p=.92, r=-.02$, teaching: $W=147.5, p=.15, r=$ -.26 , baseline: $W=136, p=.34, r=-.18)$.

\section{Study 3}

\section{Methods}

Participants and materials. We ran a pilot experiment where we assigned 13 children to the "turns condition." To determine our sample size for the current study, we ran a simulation based on data from this pilot and from the "taking over" data from our previous experiment because we expected similar effect sizes. We ran 10,000 Wilcoxon rank-sum tests with various samples bootstrapped with replacement from the initial data set. Then we coded each significant model as 1 and each insignificant model as 0 . Dividing the number of significant models by the total number of simulations gives us an estimate of our simulated studies' power. We found that the simulated power of $\mathrm{n}=30$ was .80 , so we preregistered running 30 participants per condition.

Children were excluded $(n=10)$ for the following pre-registered exclusion criteria: for experimental error $(n=9)$ and for parent interference $(n=1)$.

Procedure details. The procedures were the same as in Study 2 except for the Turns manipulation, laid out in the main text. We did not collect parent-report CCTI data for this study.

\section{Results}

Within and across conditions, children's persistence did not relate to parent education (across: $\rho(58)=.06, p=.64$, taking over: $\rho(28)=.09, p=.62$, turns: $\rho(28)=.05, p=.79$ ), race (across: $H(5)=8.85, p=.12$, taking over: $H(4)=8.80, p=.12$, turns: $H(4)=2.59, p=.63$ ), or ethnicity (across: $W=227.5, p=.88, r=-.02$, taking over: $W=69, p=.40, r=-.15$, turns: $W=$ $48.5, p=.71, r=-.07)$. There was a trend across conditions for girls persisting more than boys across conditions ( $W=565, p=.07, r=-.24$ ), but not within conditions (taking over: $W=136.5$, $p=.24, r=-.22$, turns: $W=135.5, p=.34, r=-.18)$. Although there was no significant relationship between age and children's persistence across conditions $(\rho(58)=.15, p=.26)$, or in the turns condition $(\rho(28)=-.13, p=.49)$, there was a significant positive relationship between age and children's persistence in the taking over condition $(\rho(28)=.41, p=.02)$. 
SM Table S1. Parent-Child Interaction Block Design Coding Scheme

\begin{tabular}{|c|c|c|}
\hline Behavioral Code & Definition & Example \\
\hline Encouragement & $\begin{array}{l}\text { A verbal or non-verbal } \\
\text { statement to encourage the } \\
\text { child. Positive feedback when } \\
\text { directly tied to a child's correct } \\
\text { action. }\end{array}$ & $\begin{array}{l}\text { "Keep trying," "you got this," } \\
\text { "good job,” high five, "perfect," } \\
\text { "yes," "alright" }\end{array}$ \\
\hline Quitting & $\begin{array}{l}\text { Giving up on a puzzle and } \\
\text { moving to the next one. Judge } \\
\text { quitting depending on parent } \\
\text { perception of completion. }\end{array}$ & $\begin{array}{l}\text { "Let's move on to the next one," } \\
\text { "let's leave it" }\end{array}$ \\
\hline Direct instruction & $\begin{array}{l}\text { Parent tells child exactly what } \\
\text { to do or gives child explicit } \\
\text { feedback about where things go. }\end{array}$ & $\begin{array}{l}\text { "Find a red piece," "put that } \\
\text { piece here" }\end{array}$ \\
\hline Indirect instruction & $\begin{array}{l}\text { Parent indirectly tells child } \\
\text { what to do - have child be } \\
\text { active participant/ discoverer. } \\
\text { Parent makes the task easier and } \\
\text { breaks it into chunks. }\end{array}$ & $\begin{array}{l}\text { "Find a piece that looks like this } \\
\text { (point to picture)." Parent takes } \\
\text { away all but the required blocks. } \\
\text { Parent covers up picture so child } \\
\text { just focuses on one block at a } \\
\text { time }\end{array}$ \\
\hline Taking over & $\begin{array}{l}\text { Parent completes action for } \\
\text { child — specifically moves a } \\
\text { piece. }\end{array}$ & $\begin{array}{l}\text { Parent places puzzle piece for } \\
\text { child correctly }\end{array}$ \\
\hline Pedagogical question & $\begin{array}{l}\text { Parent asks probing question } \\
\text { that they already know the } \\
\text { answer to - intended to teach } \\
\text { and help the child learn. To } \\
\text { break down problem into } \\
\text { smaller parts. }\end{array}$ & $\begin{array}{l}\text { "What piece do you think goes } \\
\text { here?" }\end{array}$ \\
\hline
\end{tabular}


SM Figure S1. Coder 1 vs. Coder 2 Scores for Parent-Child Interaction Block Design Coding.
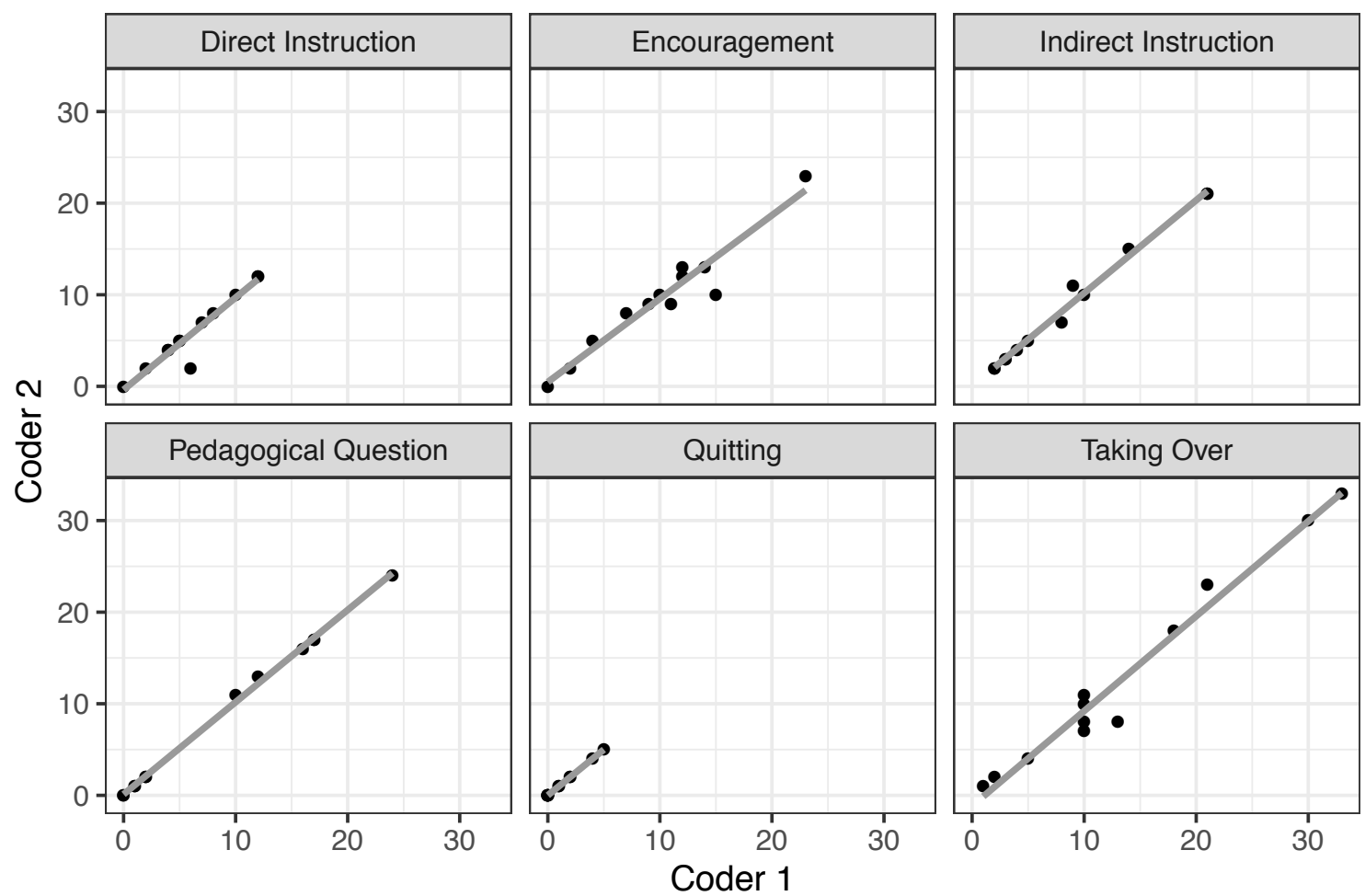